\title{
Automating and estimating glomerular filtration rate for dosing medications and staging chronic kidney disease
}

This article was published in the following Dove Press journal:

International Journal of General Medicine

2 May 2014

Number of times this article has been viewed

\author{
Katy E Trinkley' \\ $S$ Michelle Nikels ${ }^{2}$ \\ Robert L Page II' \\ Melanie S Joy' \\ 'Skaggs School of Pharmacy and \\ Pharmaceutical Sciences, ${ }^{2}$ School of \\ Medicine, University of Colorado, \\ Aurora, CO, USA
}

Correspondence: Katy E Trinkley

Department of Clinical Pharmacy, Skaggs

School of Pharmacy and Pharmaceutical

Sciences, University of Colorado

Anschutz Medical Campus, 12850 East

Montview Boulevard, Mail Stop C238,

Aurora, CO 80045, USA

$\mathrm{Tel}+\mathrm{I} 3037246563$

Fax + I 3037240979

Email katy.trinkley@ucdenver.edu
Objective: The purpose of this paper is to serve as a review for primary care providers on the bedside methods for estimating glomerular filtration rate (GFR) for dosing and chronic kidney disease (CKD) staging and to discuss how automated health information technologies (HIT) can enhance clinical documentation of staging and reduce medication errors in patients with CKD. Methods: A nonsystematic search of PubMed (through March 2013) was conducted to determine the optimal approach to estimate GFR for dosing and CKD staging and to identify examples of how automated HITs can improve health outcomes in patients with CKD. Papers known to the authors were included, as were scientific statements. Articles were chosen based on the judgment of the authors.

Results: Drug-dosing decisions should be based on the method used in the published studies and package labeling that have been determined to be safe, which is most often the CockcroftGault formula unadjusted for body weight. Although Modification of Diet in Renal Disease is more commonly used in practice for staging, the CKD-Epidemiology Collaboration (CKD-EPI) equation is the most accurate formula for estimating the CKD staging, especially at higher GFR values. Automated HITs offer a solution to the complexity of determining which equation to use for a given clinical scenario. HITs can educate providers on which formula to use and how to apply the formula in a given clinical situation, ultimately improving appropriate medication and medical management in CKD patients.

Conclusion: Appropriate estimation of GFR is key to optimal health outcomes. HITs assist clinicians in both choosing the most appropriate GFR estimation formula and in applying the results of the GFR estimation in practice. Key limitations of the recommendations in this paper are the available evidence. Further studies are needed to better understand the best method for estimating GFR.

Keywords: laboratory automation, glomerular filtration rate, medications, dose adjustment

\section{Introduction}

Accurate estimation of kidney function is essential for appropriate medical and medication management and to prevent medication errors. Common examples of medication errors in patients with kidney disease include: inappropriate drug dose adjustments for degree of kidney function; therapeutic omissions of renal protective agents; failure to monitor and adjust for chronic kidney disease (CKD) progression and subsequent changes to drug regimens; and avoidance of nephrotoxins. Approximately $23 \%$ of medications that need to be dose- or regimen-modified in CKD are not appropriately adjusted. ${ }^{1}$ Additionally, $13 \%$ of the medications given to persons with CKD are contraindicated. ${ }^{2}$ Failure to appropriately dose adjust medications for declines in kidney function significantly increases the risk of mortality by $40 \%$ and can significantly increase health 
care utilization costs. ${ }^{2}$ For example, inappropriate dose adjustment of antithrombotics can result in minor or major bleeding events, which cost more than US\$600 and US\$1,500, respectively. ${ }^{3}$ The progressive nature of kidney dysfunction warrants close vigilance to ensure that as the function declines, medications are appropriately managed.

An estimated $59 \%$ of patients with CKD are prescribed drugs that are known to be cardioprotective and/ or renoprotective. ${ }^{4}$ As even mild CKD is an independent predictor of significant cardiovascular morbidity and allcause mortality, the timely addition of cardioprotective and renoprotective agents is imperative. There is extensive evidence that demonstrates slowing the decline of kidney function $^{5-10}$ and improvement in cardiovascular outcomes ${ }^{11-12}$ with angiotensin-converting enzyme inhibitors and angiotensin receptor blockers. Preventable medication errors in patients with CKD are concerning because they not only contribute to the nearly $\$ 3$ trillion spent annually on health care expenditures in the US, but they also lead to suboptimal health and quality-of-life outcomes. The reason for these medication errors is multifactorial and includes: inappropriate estimation of kidney function; the progressive nature of kidney function decline in CKD; lack of provider time to estimate kidney function; inadequate education on how to interpret and use the various measures for estimating kidney function; and confusion regarding the use of one equation for staging kidney disease and another equation for dosing drugs. Measuring kidney function requires measurement of inulin, iothalamate, or iohexol clearance by analytical techniques and equations not readily available to clinicians. ${ }^{13}$

Estimation of the glomerular filtration rate (GFR) is performed clinically through the use of an equation that can be performed at the bedside. As GFR declines, some medications require adjustments at specific GFR thresholds, and some medications do not require dose- or regimenadjustments at all. Examples of common drugs that primary care clinicians encounter that require dose- and regimenadjustments in CKD are presented in Table 1.

Instead of using GFR, providers often inappropriately rely on serum creatinine ( $\mathrm{SCr}$ ) measurement alone to determine kidney function. SCr provides limited information, as there is significant variability between the measured $\mathrm{SCr}$ and GFR between patients. Furthermore, while declines in GFR and kidney function are in the same direction, $\mathrm{SCr}$ is reciprocal to kidney function with increasing $\mathrm{SCr}$ representing a decline in function. However, due to the busy practices of primary care clinicians, kidney function
Table I Commonly used drugs that require renal dose adjustments or are contraindicated at variable GFR thresholds ${ }^{\mathrm{a}}$

\begin{tabular}{l} 
Medications requiring dose adjustments \\
Acyclovir \\
Allopurinol \\
Amoxicillin \\
Amoxicillin/clavulanate \\
Ciprofloxacin \\
Dabigatran \\
Enoxaparin \\
Famotidine \\
Fluconazole \\
Gabapentin \\
Gemifloxacin \\
Glimepiride \\
Levetiracetam \\
Levofloxacin \\
Memantine \\
Metformin \\
Metronidazole \\
Penicillin g \\
Phenazopyridine \\
Piperacillin/tazobactam \\
Pregabalin \\
Ranitidine \\
Rivaroxaban \\
Sitagliptin \\
Sulfamethoxazole/trimethoprim \\
Tenofovir \\
Tramadol \\
Varenicline \\
Zoledronic acid \\
Contraindicated medications \\
Eplerenone \\
Exenatide \\
Glyburide \\
Liraglutide \\
Nitrofurantoin \\
Nonsteroidal anti-inflammatory drugs \\
Probenecid \\
Notone \\
\hline These
\end{tabular}

Notes: ${ }^{\text {TThese medications do not require dose adjustments and are not }}$ contraindicated for all persons with CKD. Each medication requires either a dose adjustment or is contraindicated as a unique GFR value.

Abbreviations: GFR, glomerular filtration rate; CKD, chronic kidney disease; ER, extended release.

is often mentally estimated via the SCr level versus the calculation of a GFR.

A recent study of 19 clinicians found that $79 \%$ rely on both GFR and SCr measurements when the GFR estimation is automatically reported by the laboratory; however, 100\% rely solely on $\mathrm{SCr}$ as an indicator of kidney function when GFR is not automatically reported by the laboratory. ${ }^{14}$ Some of the many factors that influence SCr include sex, age, racerelated differences in muscle mass, exercise, malnutrition, diurnal variation, cirrhosis, and drugs. Even when $\mathrm{SCr}$ is 
within the normal range, a person can have CKD. Relying on $\mathrm{SCr}$ alone results in the underdiagnosis of CKD, given that $25 \%$ of SCr measurements within normal limits translates to a GFR that is diagnostic for CKD. ${ }^{15}$ For example, based on SCr alone, a patient with an SCr of $0.9 \mathrm{mg} / \mathrm{dL}$ would not be diagnosed with $\mathrm{CKD}$; however, further assessment via GFR indicates stage $3 \mathrm{CKD}$. Table 2 summarizes the typical GFR values and measurement methods for CKD staging and drug dosing.

Many formulas have been developed to estimate GFR, and most rely on SCr measurements. In general, the GFR estimating equations have niches in clinical practice; however, the many formulas that can be used to estimate GFR make it challenging for providers to appropriately identify the ideal formula for a given situation. Further complicating the GFR estimation issue, laboratories, or health information technology (HIT) services may not automate GFR reporting, may use an incorrect formula, or may use a formula that has not been identified for the clinician.

The purpose of this manuscript is to serve as a review for primary care providers on the bedside methods for estimating GFR for dosing and CKD staging and to discuss how HIT can be used to automate the reporting process to enhance clinical documentation of staging and to reduce medication errors in patients with kidney disease.

\section{Methods}

A nonsystematic search of PubMed (through March 2013) was conducted to determine the optimal approach to estimate GFR for dosing and CKD staging and to identify examples of how automated HITs can improve health outcomes in patients with CKD. Papers known to the authors were included, as were scientific statements. Articles were chosen based on the judgment of the authors. Inherent in the design of nonsystematic reviews is author bias in article selection.

\section{Using appropriate GFR estimation formula}

Common equations for estimating GFR include: the Modification of Diet in Renal Disease (MDRD); ${ }^{16}$ the Cockcroft-Gault (CG); ${ }^{17}$ and the Chronic Kidney Disease Epidemiology Collaboration (CKD-EPI) ${ }^{18}$ Although each equation has its unique niche in practice and its own set of limitations, central to all of these equations are the incorporation of corrections for age, race, weight, sex, and other factors in addition to SCr. Body surface area (BSA) is incorporated into some equations with the thought that GFR is proportional to kidney size, which is proportional to BSA.

The CG or estimated creatinine clearance equation was the first of these three equations developed; it was determined by studying predominately hospitalized adult male patients. ${ }^{16}$ The $\mathrm{CG}$ equation attempts to control for age, sex, and weight; it is reported as $\mathrm{mL} / \mathrm{min} .{ }^{16}$ The $\mathrm{CG}$ equation is often the standard equation used for calculating drug doses, since historical drug labeling contains dosing information in reference to CG. However, the CG equation underestimates GFR in the elderly and is less accurate in patients with normal kidney function.

The MDRD equation (abbreviated as "eGFR") was the second equation developed. It was developed by studying nonhospitalized persons with CKD. ${ }^{17}$ MDRD normalizes for race, $\mathrm{BSA}$, age, and sex; it is reported as $\mathrm{mL} / \mathrm{minute} / 1.73 \mathrm{~m}^{2}$. MDRD is predominantly used for CKD staging in clinical practice. Several limitations of the MDRD equation have been documented. Since the MDRD equation was derived from persons with CKD, it is imprecise and underestimates GFR at higher values, yielding false positives for CKD. Furthermore,

Table 2 Methods for assessing kidney function by GFR and staging CKD

\begin{tabular}{|c|c|c|}
\hline Formula for assessing GFR & Purpose & Interpretation by GFR ${ }^{\mathbf{a}}$ \\
\hline MDRD $^{16}$ & Staging CKD & CKD stage I: GFR $>90 \mathrm{~mL} /$ minute $/ \mathrm{l} .73 \mathrm{~m}^{2}$ \\
\hline \multirow[t]{4}{*}{ CKD-EPI ${ }^{17}$} & Staging CKD & CKD stage 2: GFR $60-89 \mathrm{~mL} /$ minute/ $1.73 \mathrm{~m}^{2}$ \\
\hline & & CKD stage 3: GFR 30-59 mL/minute/l.73 $\mathrm{m}^{2}$ \\
\hline & & CKD stage 4: GFR I5-29 mL/minute/l.73 m² \\
\hline & & CKD stage 5: GFR < $15 \mathrm{~mL} /$ minute/ $1.73 \mathrm{~m}^{2}$ \\
\hline \multirow[t]{6}{*}{ Cockcroft-Gault $(\mathrm{CG})^{18}$} & Dose adjustment & Degree of dose adjustment varies by drug and CG-estimated GFR \\
\hline & & Normal renal function: $>80 \mathrm{~mL} /$ minute \\
\hline & & Mild impairment: $50-80 \mathrm{~mL} / \mathrm{minute}$ \\
\hline & & Moderate impairment: $30-50 \mathrm{~mL} /$ minute \\
\hline & & Severe impairment: $<30 \mathrm{~mL} /$ minute \\
\hline & & End stage renal disease: dialysis required ${ }^{5}$ \\
\hline
\end{tabular}

Note: ${ }^{\text {aCKD }}$ staging also includes evidence of kidney damage, which is not included in this table.

Abbreviations: GFR, glomerular filtration rate; CKD, chronic kidney disease; MDRD, Modification of Diet in Renal Disease. 
the MDRD equation has not been evaluated in: persons $<18$ years of age; persons $>75$ years of age; pregnant women; extremes in body size; or in races other than Caucasian and African American. ${ }^{17}$

CKD-EPI, the newest equation, was determined with a cross-sectional analysis of a sample of persons who were representative of the US population. ${ }^{18}$ The CKD-EPI normalizes for race, $\mathrm{BSA}$, age, and sex and is reported as $\mathrm{mL} / \mathrm{min} / 1.73 \mathrm{~m}^{2}$. In clinical practice, CKD-EPI is most commonly used for CKD staging. Although the CKD-EPI is a more accurate estimation of GFR than MDRD at all values and more representative of the US population, the CKD-EPI sample population included limited elderly and minority populations. ${ }^{18}$ To compare the MDRD or CKD-EPI values with CG, it is customary to multiply or to adjust the MDRD and CKD-EPI values (mL/minute/1.73 $\mathrm{m}^{2}$ ) by the patient's BSA to have all equation results in the same units of measurement ( $\mathrm{mL} /$ minute). Table 3 describes the MDRD, CG, and CKD-EPI formulas and the applicable factors in each equation.

Each equation incorporates $\mathrm{SCr}$. However, in recent years, all SCr measurements in the US became standardized to prevent variations due to the assay or instrument used, which can impact the accuracy of the equations if not taken into account. Of the commonly used GFR estimation formulas, the CKD-EPI was actually developed using the standardized SCr measurements, while the MDRD equation was reexpressed for use with the standardized SCr measurements. Unfortunately, CG cannot be re-expressed, because the original blood samples used to develop the $\mathrm{CG}$ equation are no longer available. As a result of not being able to re-express the $\mathrm{CG}$ equation for standardized SCr, CG-estimated GFR results are 5\%-10\% higher using the standardized SCr measurements when compared to nonstandardized SCr. ${ }^{19}$ Relying on CG-estimated GFR based on standardized SCr measurements decreases the accuracy of this GFR estimation and could lead to unintended consequences, including insufficient dose adjustments for kidney function. However, the clinical significance of this theoretical issue requires study.

\section{Estimating GFR for medication adjustment}

Appropriately adjusting a medication dose or regimen for kidney function ensures the medication reaches safe and effective drug concentrations for the targeted indication and ultimately achieves optimal clinical outcomes. Therefore, appropriate drug dose- and regimen-adjustments are imperative to the provision of quality health care. However, the use of several estimating equations for kidney function assessment can be confusing for clinicians who need to discern which equation should be used for dosing medications.

The CG equation is the most often used formula for doseadjusting medications based on kidney function and is endorsed by the National Kidney Foundation (NKF) ${ }^{20}$ and the American College of Cardiology (ACC)/American Heart Association (AHA). ${ }^{21}$ However, the NKF also recommends that - in addition to $\mathrm{CG}$ - the MDRD unadjusted for BSA (not multiplying MDRD by the patient's BSA) is a reasonable method for adjusting medication doses based on kidney function. ${ }^{20}$

It is important to discriminate that while the NKF and the ACC/AHA have more leverage regarding clinical practice issues, the US Food and Drug Administration (FDA) has less impact on clinical practice but extensive oversight on the pharmaceutical industry and drug development process. Despite some inconsistency in the national recommendations as to which formula to use for drug dose and regimen adjustments in CKD, the 1998 FDA Guidance for Industry document recommends using $\mathrm{CG}$ for drug labeling recommendations. ${ }^{22}$ However, a 2010 updated draft of the 1998 FDA document incorporates reporting both CG and MDRD GFR estimates in the literature of new products, ${ }^{23}$ but the draft has not yet been finalized. It is important to understand that most recommendations in the drug product

Table 3 Common GFR estimation formulas

\begin{tabular}{|c|c|c|}
\hline Cockcroft-Gault (eCrCl) $)^{16}$ & \multicolumn{2}{|c|}{$([140-$ age in years $] \times$ ideal body weight in $\mathrm{kg})(\times 0.85$ if female $)([\mathrm{SCr}$ in $\mathrm{mg} / \mathrm{dL}] \times 72)$} \\
\hline MDRD (eGFR) ${ }^{17}$ & \multicolumn{2}{|c|}{$170 \times(\mathrm{SCr} \text { in } \mathrm{mg} / \text { day })^{-0.999} \times(\text { age in years })^{0.318} \times(0.762$ if female $) \times(1.18$ if African American $)$} \\
\hline \multirow[t]{8}{*}{ CKD-EPI ${ }^{18}$} & African American, female, $\mathrm{SCr} \leq 0.7$ & $166 \times([\mathrm{SCr} \text { in } \mathrm{mg} / \mathrm{dL}] / 0.7)^{-0.329} \times(0.993)^{\text {(age in years) }}$ \\
\hline & African American, female, $\mathrm{SCr}>0.7$ & $166 \times([\mathrm{SCr} \text { in } \mathrm{mg} / \mathrm{dL}] / 0.7)^{-1.209} \times(0.993)^{\text {(age in years) }}$ \\
\hline & African American, male, $\mathrm{SCr} \leq 0.9$ & $163 \times([\mathrm{SCr} \text { in } \mathrm{mg} / \mathrm{dL}] / 0.9)^{-0.411} \times(0.993)^{\text {(age in years })}$ \\
\hline & African American, male, $\mathrm{SCr}>0.9$ & $163 \times([\mathrm{SCr} \text { in } \mathrm{mg} / \mathrm{dL}] / 0.9)^{-1.209} \times(0.993)^{\text {(age in years })}$ \\
\hline & White or other, female, $\mathrm{SCr} \leq 0.7$ & $144 \times([\mathrm{SCr} \text { in } \mathrm{mg} / \mathrm{dL}] / 0.7)^{-0.329} \times(0.993)^{(\text {age in years })}$ \\
\hline & White or other, female, $\mathrm{SCr}>0.7$ & $144 \times([\mathrm{SCr} \text { in } \mathrm{mg} / \mathrm{dL}] / 0.7)^{-01.209} \times(0.993)^{\text {(age in years) }}$ \\
\hline & White or other, female, $\mathrm{SCr} \leq 0.9$ & $14 \mathrm{I} \times([\mathrm{SCr} \text { in } \mathrm{mg} / \mathrm{dL}] / 0.7)^{-0.411} \times(0.993)^{(\text {age in years })}$ \\
\hline & White or other, female, $\mathrm{SCr}>0.9$ & $14 \mathrm{I} \times([\mathrm{SCr} \text { in } \mathrm{mg} / \mathrm{dL}] / 0.7)^{-1.209} \times(0.993)^{\text {(age in years) }}$ \\
\hline
\end{tabular}

Abbreviations: $\mathrm{eCrCl}$, Cockcroft-Gault equation; GFR, glomerular filtration rate; eGFR, glomerular filtration rate; CKD-EPI, CKD-Epidemiology Collaboration equation; $\mathrm{SCr}$, serum creatinine; CKD, chronic kidney disease; MDRD, Modification of Diet in Renal Disease. 
literature are based on studies using the $\mathrm{CG}$ equation, ${ }^{22}$ and many of these recommendations are based on $\mathrm{CG}$ before the implementation of standardized SCr. Because drug labeling recommendations for safe and effective dose and regimen adjustments are based on the CG equation, it is customary to use $\mathrm{CG}$ for drug-dosing decisions.

Although the current FDA Guidance for Industry recommends the use of CG when designing studies, it does not provide guidance on whether CG should be adjusted for body weight. As a consequence, drug labels usually do not indicate information about whether GFR was adjusted for weight. A systematic review of drug labels approved from 1998-2007 identified 44 labels that recommended dose adjustments based on kidney function; however, only eleven specified that $\mathrm{CG}$ should be used and adjusting for body weight was not specified in six of the eleven labels. ${ }^{24}$ The original CG formula, published in 1976, used the patient's actual versus adjusted or ideal body weight. Later applications of CG most often adjust for ideal body weight, given the higher body weights of today versus those in 1976. Interestingly, CG adjusted for ideal body weight is less accurate at estimating GFR than unadjusted weight. ${ }^{5,25-28} \mathrm{CG}$ accurately estimates GFR $66 \%$ of the time when adjusted for ideal body weight, compared to $73 \%$ of the time when using actual body weight. ${ }^{37}$ When using CG for dosing decisions, the decision to adjust for body weight should be based on the method used in the studies that determined the dose adjustment recommendations.

Several studies have compared MDRD with CG for dosing medications. One study evaluated 15 FDA-approved drugs and determined the actual GFR for 5,000 subjects. The study found that MDRD adjusted for BSA (reported as $\mathrm{mL} /$ minute) correctly identified dose reductions $88 \%$ of the time, while the $\mathrm{CG}$ equation accurately calculated the renal dose adjustments $85 \%$ and $82 \%$ of the time, using actual and ideal body weights, respectively. ${ }^{27}$

In contrast, data from the Can Rapid risk stratification of Unstable angina patients Suppress ADverse outcomes with Early implementation of the ACC/AHA guidelines (CRUSADE) compared CG using ideal body weight with MDRD adjusted for BSA for dosing dose-adjusting antithrombotics for kidney function. ${ }^{29}$ This study found that CG led to $43 \%$ more cases of antithrombotic dose adjustments than MDRD (30,386 versus 17,329 dose adjustments with CG and MDRD, respectively). Major bleeding occurred in $17.8 \%$ and $21.8 \%$ of patients who received excessive antithrombotic doses as determined by the CG and MDRD formula, respectively. Minor bleeding events were not reported. Given the narrow therapeutic index of the antithrombotics, the risks of bleeding and increased hospital stays are elevated if the dose is not appropriately adjusted for kidney function. Despite these risks, as well as drug-product labeling and national recommendations to dose adjust antithrombotics based on $\mathrm{CG},{ }^{21}$ $87 \%$ of hospitals do not follow the drug-labeling recommendations for renal dose adjustments. ${ }^{30}$ Another study evaluated dose adjustments in kidney dysfunction for digoxin, another narrow therapeutic index drug. Similar to the results of CRUSADE, this study found that the CG unadjusted for body weight led to $32 \%$ more dose reductions of digoxin than MDRD unadjusted for BSA. ${ }^{31}$

Although there are several studies comparing MDRD to CG for drug doses and adverse events, no clinical trials have compared the clinical outcomes of dose and regimen adjustments for patients with CKD on the basis of $\mathrm{CG}$ adjusted for ideal body weight compared to unadjusted MDRD. Until more information is available from GFR calculating methods on a comprehensive list of drugs and/or information is available that demonstrates the clinical outcomes associated with these dosing methods, clinicians should dose adjust medications for kidney function based on the methods used in the published studies and package labeling that have been determined to be safe.

\section{Estimating GFR for staging CKD}

Correctly diagnosing and staging CKD is imperative to add appropriate nephroprotective agents and nondrug management to the regimen, to improve vigilance in avoiding nephrotoxic agents, and to screen for and initiate therapies for the common complications of $\mathrm{CKD}$, such as anemia, derangements in electrolytes, and mineral metabolism disorders.

The two most common equations for staging CKD are the MDRD and the CKD-EPI. There is controversy over whether the MDRD or the CKD-EPI is the most appropriate method for estimating GFR for CKD diagnosis, which may stem from a limited knowledge of the new CKD-EPI formula and of the evidence supporting its superiority to MDRD. While MDRD is commonly used for estimating GFR, CKD-EPI is gaining momentum as the optimal GFR estimating equation. In fact, the NKF now recognizes the CKD-EPI formula as more accurate than the MDRD equation, based on head-to-head comparisons of the two formulas..$^{20}$ The CKD-EPI equation was specifically developed to overcome the limitations of the MDRD equation.

As a result of differences in study populations used to define the GFR estimating equations, the CKD-EPI equation is as accurate as the MDRD at GFR $<60 \mathrm{~mL} /$ minute and more 
accurate than MDRD at GFR $>60 \mathrm{~mL} /$ minute. The superior accuracy of CKD-EPI across the ranges of GFR has led to a lower estimated prevalence of CKD than that reported with the MDRD (11.5\% versus $13.1 \%)$. Additionally, more reclassifications of CKD staging to less-severe CKD stages have been reported for patients in the $30-59 \mathrm{~mL} /$ minute $/ 1.73 \mathrm{~m}^{2}$ range of GFR (stage 3 CKD).

For example, patients who were originally classified as stage 3 CKD with MDRD may be reclassified as stage 2 by the CKD-EPI equation. When comparing the discordance of CKD staging between MDRD and CKD-EPI, classification by CKD-EPI was correct $65 \%$ of the time, compared to MDRD, which was correct only $34 \%$ of the time $(P<0.001) .^{18}$

Relying on the MDRD to screen for CKD decreases the sensitivity and specificity of identifying persons who are near the GFR threshold of $60 \mathrm{~mL} /$ minute $/ 1.73 \mathrm{~m}^{2}$ (stage $3 \mathrm{CKD}$ ) and decreases the likelihood that CKD staging is accurate. Hence, CKD-EPI is preferred for identifying patients with $\mathrm{CKD}$ and for staging the disease. The risk of underestimation of kidney function with MDRD is highest when the GFR is $>30 \mathrm{~mL} /$ minute $/ 1.73 \mathrm{~m}^{2}$. Therefore, it is especially important to calculate the CKD-EPI for these persons. Using CKD-EPI for diagnosis and staging may more accurately be recommended when the addition of appropriate prophylactic drugs or avoidance of certain nephrotoxic drugs should occur.

\section{Automation of GFR and HIT}

When GFR estimation is automated, the GFR is reported any time that $\mathrm{SCr}$ is ordered by clinicians. Although not all laboratories or HIT services automate GFR estimation, there are many benefits to automating GFR estimation. Automation of GFR estimation improves: detection of CKD; appropriate referral to nephrology services; provider reliance on GFR versus SCr alone; and, ultimately, clinical outcomes. Implementation of automated GFR estimation significantly improves identification of CKD by nearly $50 \%$. It also can result in blood pressure goal attainment and the addition of nephroprotective angiotensin-converting enzyme inhibitors or angiotensin receptor blockers to therapy. While the improvements in blood pressure and the addition of nephroprotective agents were statistically significant, the relative percent improvements in achieving these endpoints were found to be modest at $3 \%$ and $4.6 \%$, respectively. This may suggest that despite incorporation of HIT services, widespread outcome improvements are not automatic due to patient-related factors, including compliance. Automation can also improve referrals to nephrology by $40 \% .{ }^{32,33}$ Most importantly, it has been reported that automation of GFR estimation assists in decreasing the decline in GFR from $3.69 \mathrm{~mL} /$ minute $/ 1.73 \mathrm{~m}^{2}$ during the 9 months prior to automation to $0.32 \mathrm{~mL} /$ minute $/ 1.73 \mathrm{~m}^{2}$ during the 12 months postimplementation $(P<0.001) .{ }^{34}$ The reduction in kidney function decline could have huge benefits for health care costs.

Because of the many benefits of automated GFR estimation, automation is becoming standard of practice. Currently, most laboratories and HIT services that report automated GFR use the MDRD equation. It is important to realize that MDRD reporting may be underestimating the actual GFR. There are many GFR estimating equations and as automation becomes the standard, the automation process needs to clearly identify which GFR formula was coded and reported. Additionally, the variables used in the formula (ideal body weight versus actual body weight) and units of the GFR result ( $\mathrm{mL} /$ minute versus $\mathrm{mL} /$ minute $/ 1.73 \mathrm{~m}^{2}$ ) need to be clearly identified. Furthermore, it may be useful for automation to include reporting more than one GFR estimation formula, given no one formula is appropriate for all clinical situations. Reporting both CG and either MDRD or CKD-EPI would enhance drugdosing adjustments as well as enable CKD staging. Choosing the appropriate method for determining GFR is challenging, given the multiple formulas, varying patient care situations in which GFR is used and the complexity of determining the method of kidney function estimation used in the original drug studies or recommended by drug labels. Using clinical decision-support tools within an HIT service that automates GFR estimation could serve a dual purpose to educate providers of which formula is most appropriate to use and how to apply the formula in a given clinical situation.

Within HIT services, it is possible to automatically report estimated GFR using several equations and highlight when each should be used based on the clinical situation. For example, while ordering or refilling dabigatran for a patient with an estimated GFR of $29 \mathrm{~mL} /$ minute, the HIT would be programmed with decision support that would pop-up and educate the provider that the dose of dabigatran needs to be reduced or an alternate anticoagulant initiated, because of the patient's estimated GFR. Such decision-support tools are especially helpful for drugs with narrow therapeutic indexes, such as the new oral anticoagulants (dabigatran, enoxaparin, apixaban) and older injectable antithrombotics (enoxaparin) that require renal dose adjustments to prevent bleeding events.

Appropriate dose adjustments for the new oral anticoagulants are especially imperative, because there is currently no reversal agent in the event of excess dose or bleeding. Another such decision-support tool may be a best practice alert that 
pops up during an outpatient encounter with a patient who has a CKD-EPI estimated GFR of $58 \mathrm{~mL} /$ minute $/ 1.73 \mathrm{~m}^{2}$, and who is not on appropriate renoprotective and cardioprotective agents, such as an angiotensin-converting enzyme inhibitor. This decision-support tool educates the provider at the point of care that the patient should be on certain prophylactic medications, unless there are contraindications.

HIT is increasingly being used for automated GFR estimation reporting. However, there are limitations to the automation of GFR reporting, including inaccurate coding of the equations in the HIT, lack of reference to the equation(s) being used, lack of understanding of the reference equation's clinical limitations, and incorrect interpretation and application of the result. Currently, automation of GFR estimation is typically only reported as MDRD adjusted for BSA, despite the feasibility of incorporating automated reporting of CKD-EPI at most institutions. Automated reporting of MDRD instead of CKD-EPI could contribute to an overdiagnosis of CKD.

\section{Conclusion}

Although there are divergent opinions regarding the best GFR estimation equation to use for the staging of CKD and the dosing of medications, most current data support CKD-EPI as the most accurate method for diagnosis and staging of CKD and $\mathrm{CG}$ for drug-dosing decisions. Historically, drug labeling recommendations are based on the $\mathrm{CG}$ equation. However, the FDA is considering recommending that both CG and MDRD be incorporated into the drug label. ${ }^{23}$ Despite some current confusion regarding GFR estimating equations for CKD staging as opposed to drug dosing, one central element that clinicians should take from this review is that $\mathrm{SCr}$ measurements alone should never be used for estimating kidney function. Clinicians need to ensure that HIT services at their institutions convey the necessary level of detail regarding the equation used to report estimated GFR (MDRD versus CKD-EPI versus CG) and the education surrounding the limitations of the estimating equation used. Appropriate estimation of GFR through HIT can improve health outcomes, improve patient safety, and decrease unnecessary health care expenditures.

\section{Disclosure}

The authors report no conflicts of interest in this work.

\section{References}

1. Frölich T, Zorina O, Fontana AO, Kullak-Ublick GA, Vollenweider A, Russmann S. Evaluation of medication safety in the discharge medication of 509 surgical inpatients using electronic prescription support software and an extended operational interaction classification. Eur J Clin Pharmacol. 2011;67(12):1273-1282.
2. Breton $\mathrm{G}$, Froissart M, Janus $\mathrm{N}$, et al. Inappropriate drug use and mortality in community-dwelling elderly with impaired kidney function - the Three-City population-based study. Nephrol Dial Transplant. 2011;26(9):2852-2859.

3. Gould MK, Dembitzer AD, Sanders GD, Garber AM. Low-molecularweight heparins compared with unfractionated heparin for treatment of acute deep venous thrombosis: A cost-effectiveness analysis. Ann Int Med. 1999;130(10):789-799.

4. Wyatt C, Konduri V, Eng J, Rohatgi R. Reporting of estimated GFR in the primary care clinic. Am J Kidney Dis. 2007;49(5):634-641.

5. Kshirsagar AV, Joy MS, Hogan SL, Falk RJ, Colindres RE. Effect of ACE inhibitors in diabetic and nondiabetic chronic renal disease: a systematic overview of randomized placebo-controlled trials. Am J Kidney Dis. 2000;35(4):695-707.

6. Brenner BM, Cooper ME, de Zeeuw D, et al; RENAAL Study Investigators. Effects of losartan on renal and cardiovascular outcomes in patients with type 2 diabetes and nephropathy. $N$ Engl $\mathrm{J} \mathrm{Med}$. 2001;345(12):861-869.

7. Hou FF, Zhang X, Zhang GH, et al. Efficacy and safety of benazepril for advanced chronic renal insufficiency. $N$ Engl J Med. 2006;354(2): $131-140$.

8. Ruggenenti P, Perna A, Gherardi G, Gaspari F, Benini R, Remuzzi G. Renal function and requirement for dialysis in chronic nephropathy patients on long-term ramipril: REIN follow-up trial. Gruppo Italiano di Studi Epidemiologici in Nefrologia (GISEN). Ramipril Efficacy in Nephropathy. Lancet. 1998;352(9136):1252-1256.

9. Maschio G, Alberti D, Janin G, et al; The Angiotensin-ConvertingEnzyme Inhibition in Progressive Renal Insufficiency Study Group. Effect of the angiotensin-converting-enzyme inhibitor benazepril on progression of chronic renal insufficiency. $N$ Engl J Med. 1996;334(15):939-945.

10. Ruggenenti P, Perna A, Gherardi G, et al. Renoprotective properties of ACE-inhibitors in non-diabetic nephropathies with non-nephrotic proteinuria. Lancet. 1999;345(9176):359-364.

11. de Zeeuw D, Remuzzi G, Parving HH, et al. Albuminuria, a therapeutic target for cardiovascular protection in type 2 diabetic patients with nephropathy. Circulation. 2004;110(8):921-927.

12. Yusuf S, Sleight P, Pogue J, Bosch J, Davies R, Dagenais G; The Heart Outcomes Prevention Evaluation Study Investigators. Effects of an angiotensin-converting-enzyme inhibitor, ramipril, on cardiovascular events in high-risk patients. $N$ Engl J Med. 2000;342(3):145-153.

13. Stevens LA, Levey AS. Measured GFR as a confirmatory test for estimated GFR. J Am Soc Nephrol. 2009;20(11):2305-2313.

14. Smith DH, Schneider J, Thorp ML, et al. Clinician's use of automated reports of estimated glomerular filtration rate: a qualitative study. $B M C$ Nephrol. 2012;13:154.

15. Coresh J, Astor BC, Greene T, Eknoyan G, Levey AS. Prevalence of chronic kidney disease and decreased kidney function in the adult US population: Third National Health and Nutrition Examination Survey. Am J Kidney Dis. 2003;41(1):1-12.

16. Cockcroft DW, Gault MH. Prediction of creatinine clearance from serum creatinine. Nephron. 1976;16(1):31-41.

17. Levey AS, Bosch JP, Lewis JB, Greene T, Rogers N, Roth D, et al; Modification of Diet in Renal Disease Study Group. A more accurate method to estimate glomerular filtration rate from serum creatinine: a new prediction equation. Ann Intern Med. 1999;130(6): $461-470$.

18. Levey AS, Stevens LA, Schmid CH, et al; CKD-PI (Chronic Kidney Disease Epidemiology Collaboration). A new equation to estimate glomerular filtration rate. Ann of Intern Med. 2009;150(9):604-612.

19. Miller WG, Myers GL, Ashwood ER, et al. Creatinine measurement: state of the art in accuracy and interlaboratory harmonization. Arch Pathol Lab Med. 2005;129(3):297-304.

20. National Kidney Foundation. Frequently Asked Questions About GFR Estimates. New York: National Kidney Foundation; 2011. Available from: http://www.kidney.org/professionals/kls/pdf/12-10-4004_KBB_ FAQs_AboutGFR-1.pdf. Accessed April 12, 2013. 
21. Braunwald E, Antman EM, Beasley JW, et al; American College of Cardiology; American Heart Association. Committee on the Management of Patients With Unstable Angina. ACC/AHA 2002 guideline update for the management of patients with unstable angina and non-ST-segment elevation myocardial infarction - summary article: a report of the American College of Cardiology/American Heart Association task force on practice guidelines (Committee on the Management of Patients With Unstable Angina). J Am Coll Cardiol. 2002;40(7):1366-1374.

22. US Department of Health and Human Services. Guidance for Industry: Pharmacokinetics in Patients with Impaired Renal Function - Study Design, Data Analysis, and Impact on Dosing and Labeling. Silver Spring, MD: US Food and Drug Administration; 1998. Available from: http://www.fda.gov/downloads/Drugs/GuidanceComplianceRegulatory Information/Guidances/ucm072127.pdf. Accessed February 2, 2013.

23. US Department of Health and Human Services. Guidance for Industry: Pharmacokinetics in Patients with Impaired Renal Function - Study Design, Data Analysis, and Impact on Dosing and Labeling. Silver Spring, MD: US Food and Drug Administration; 2010. Available from: http://www.fda.gov/downloads/Drugs/GuidanceComplianceRegulat oryInformation/Guidances/UCM204959.pdf. Accessed February 24, 2013.

24. Dowling TC, Matzke GR, Murphy JE, Burckart GJ. Evaluation of renal drug dosing: prescribing information and clinical pharmacist approaches. Pharmacotherapy. 2010;30(8):776-786.

25. Gonwa TA, Jennings L, Mai ML, Stark PC, Levey AS, Klintmalm GB. Estimation of glomerular filtration rates before and after orthotopic liver transplantation: evaluation of current equations. Liver Transpl. 2004;10(2):301-309.

26. O’Meara E, Chong KS, Gardner RS, Jardine AG, Neilly JB, McDonagh TA. The Modification of Diet in Renal Disease (MDRD) equations provide valid estimations of glomerular filtration rates in patients with advanced heart failure. Eur J Heart Fail. 2006;8(1):63-67.
27. Stevens LA, Nolin TD, Richardson MM, et al; Chronic Kidney Disease Epidemiology Collaboration. Comparison of drug dosing recommendations based on measured GFR and kidney function estimating equations. Am J Kidney Dis. 2009;54(1):33-42.

28. Park EJ, Pai MP, Dong T, et al. The influence of body size descriptors on the estimation of kidney function in normal weight, overweight, obese, and morbidly obese adults. Ann Pharmacother. 2012;46(3): 317-328.

29. Melloni C, Peterson ED, Chen AY, et al. Cockcroft-Gault versus modification of diet in renal disease: importance of glomerular filtration rate formula for classification of chronic kidney disease in patients with non-ST-segment elevation acute coronary syndromes. $J$ Am Coll Cardiol. 2008;51(10):991-996.

30. Barras MA, Kirkpatrick CM, Green B. Current dosing of low-molecularweight heparins does not reflect licensed product labels: an international survey. Br J Clin Pharmacol. 2010;69(5):520-528.

31. Gill J, Malyuk R, Djurdjev O, Levin A. Use of GFR equations to adjust drug doses in an elderly multi-ethnic group - a cautionary tale. Nephrol Dial Transplant. 2007;22(10):2894-2899.

32. Noble E, Johnson DW, Gray N, et al. The impact of automated eGFR reporting and education on nephrology service referrals. Nephrol Dial Transplant. 2008;23(12):3845-3850.

33. Richards N, Harris K, Whitfield M, et al. The impact of population-based identification of chronic kidney disease using estimated glomerular filtration rate (eGFR) reporting. Nephrol Dial Transplant. 2008;23(2): 556-561.

34. Richards N, Harris K, Whitfield M, et al. Primary care-based disease management of chronic kidney disease (CKD), based on estimated glomerular filtration rate (eGFR) reporting, improves patient outcomes. Nephrol Dial Transplant. 2008;23(2):549-555.
International Journal of General Medicine

\section{Publish your work in this journal}

The International Journal of General Medicine is an international, peer-reviewed open-access journal that focuses on general and internal medicine, pathogenesis, epidemiology, diagnosis, monitoring and treatment protocols. The journal is characterized by the rapid reporting of reviews, original research and clinical studies across all disease areas.

\section{Dovepress}

A key focus is the elucidation of disease processes and management protocols resulting in improved outcomes for the patient.The manuscript management system is completely online and includes a very quick and fair peer-review system. Visit http://www.dovepress.com/ testimonials.php to read real quotes from published authors. 